\title{
Invited Discussion on: Anthropometry Analysis of Beautiful Upper Eyelids in Oriental: New Eyelid Crease Ratio and Clinical Application
}

\author{
Li Y $\mathbf{U}^{1}$
}

Received: 8 December 2019/Accepted: 10 December 2019/Published online: 8 January 2020

(C) Springer Science+Business Media, LLC, part of Springer Nature and International Society of Aesthetic Plastic Surgery 2020

Level of Evidence $V$ This journal requires that authors assign a level of evidence to each article. For a full description of these Evidence-Based Medicine ratings, please refer to the Table of Contents or the online Instructions to Authors www.springer.com/00266.

The author performed anthropometry analysis of beautiful upper eyelids in Orientals and concluded a new eyelid crease ratio and its clinical application. The results show that the vertical distance of palpebral fissure, height of double fold, height of lid crease and height of closed upper eyelid were statistically significant between the most, average and less beautiful upper eyelids in both males and females. Meanwhile, the author believes that Type 1 epicanthal folds are the most common in the most beautiful upper eyelid group. The author concluded that the most beautiful double upper eyelids in Orientals demonstrated a higher vertical palpebral fissure, double eyelid fold and height of eyelid crease than average and less one. The ratio of the distance from the eyelid crease to the lower margin of the eyebrow to the height of the eyelid crease is 1.2:1 to create beautiful double upper eyelids. The author emphasizes that preoperative consultation with realistic expectations is important to plastic surgeons.

As we all know, no matter in which Asian countries, upper eyelid surgery is one of the most common aesthetic surgeries in Asia. Anatomically, Asian upper eyelids are quite different from Caucasian eyelids not only in the upper eyelid region but also in the periorbital region. In the upper

\section{Li YU}

yuoli@163.com

1 Department of Plastic and Reconstructive Surgery, Shanghai 9th People's Hospital, Shanghai Jiaotong University, School of Medicine, Shanghai, China eyelid, we can find some differences including more subcutaneous fat, thicker orbicularis oculi, puffy upper eyelids with lower fusion of the orbital septum on and even lower than the tarsal border and more pretarsal fat component. In the periorbital region, we can also find differences including more prominence of the orbital bone with a more sunken appearance of both eyes in Caucasians. All of these anatomically different appearances in different populations may gradually establish different racial esthetics in past history.

We believe the aesthetic standard of the eyes should be based on two vectors. First of a all, it is horizontal vector. Estheticians in ancient China mentioned the theory in facial aesthetic standard called: San Ting Wu Yan. This theory with the same meaning as that in the West is that the face could be divided into three vertical parts by two horizontal lines. The face is divided into five facial parts by vertical lines with one part equal to the width of palpebral fissure. This strongly indicates that the two eyes are one of the most important organs in facial aesthetics. Meanwhile, it manifests that the horizontal width of the eyes is very critical in the aesthetic standard. The horizontal width of the eyes is not only one of the targets of eye aesthetics but also should balance with the width of the face. Influenced by modern cartoon figures, lots of young female patients want to greatly enlarge the eye width with the performance of epicanthoplasty on two directions. Even in some extreme situations, the surgical opening of the lateral canthus is strongly required, although it is believed that such a surgical technique could not produce nice and nature results, on the contrary, leaving untreated deformities. The other point related to the horizontal vector of upper eyelid aesthetics is the longevity of the upper eyelid crease. In good-looking congenital double eyelids, most double 
eyelid lines, at the nasal side, do not extend through the vertical line of the inner canthus point. But all the double eyelid lines extend to the temporal side beyond the vertical line of the outer canthus point. The general characteristics of congenital double eyelids will be helpful to cosmetic surgeons and those seeking beauty for important reference. In the design of double eyelid surgery, cosmetic surgeons should be aware of this phenomenon and take reference for designing double eyelid lines.

Second is the aesthetic standard of the vertical vector. From the traditional criteria of beauty in China and the Fresco found in Dunhuang Mogao Grottoes which is the eastern pearl of art (Fig. 1), the painting has strongly implied the traditional characteristics of eye appearance. The typical characteristics of female eyes in China are the following: small willow-leaf eyes, single eyelid with small fissure, wider distance of both eyes with epicanthus, bulging upper eyelid. All of these figures are common and well acceptable by the society at that time. That is one of the reasons why we find the artistic figures are like this in so many paintings and sculptures (Fig. 2). However, as time goes by, influenced by Western-Eastern culture communication and social media broadcasting, especially after the second World War, with the recovery of the global economy and the deepening of personal communications between countries and regions, double eyelid plasty which is regarded as Westernized upper blepharoplasty first probably became popular among Asians living in Europe and the USA, and gradually was accepted and even pursued

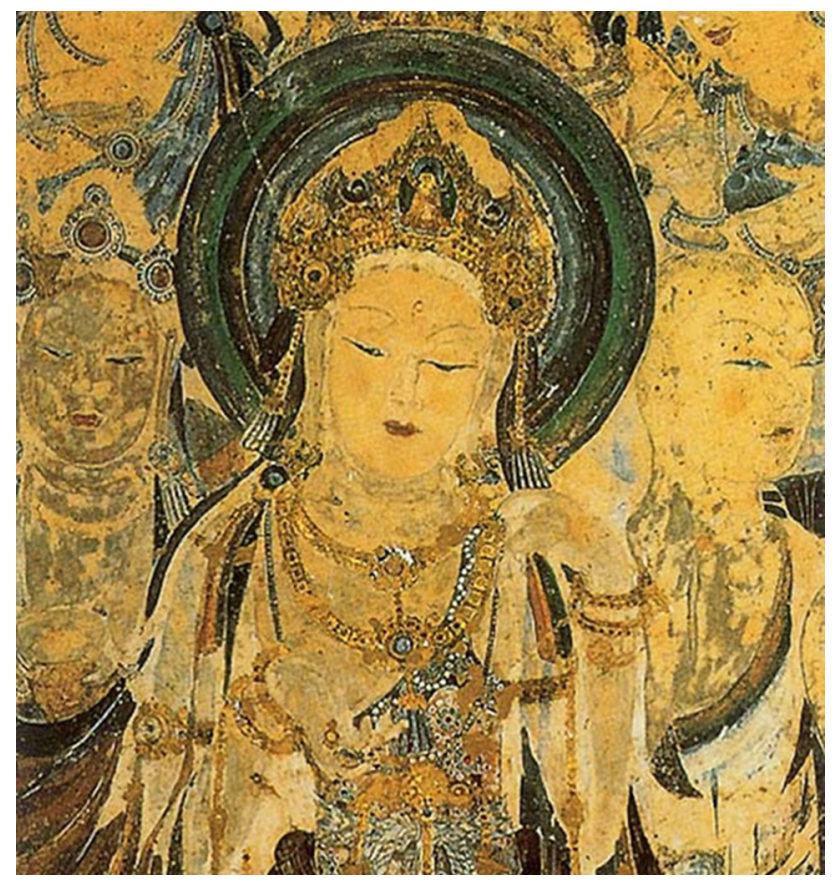

Fig. 1 The Fresco found in Dunhuang Mogao Grottoes which is one of the eastern pearl of art

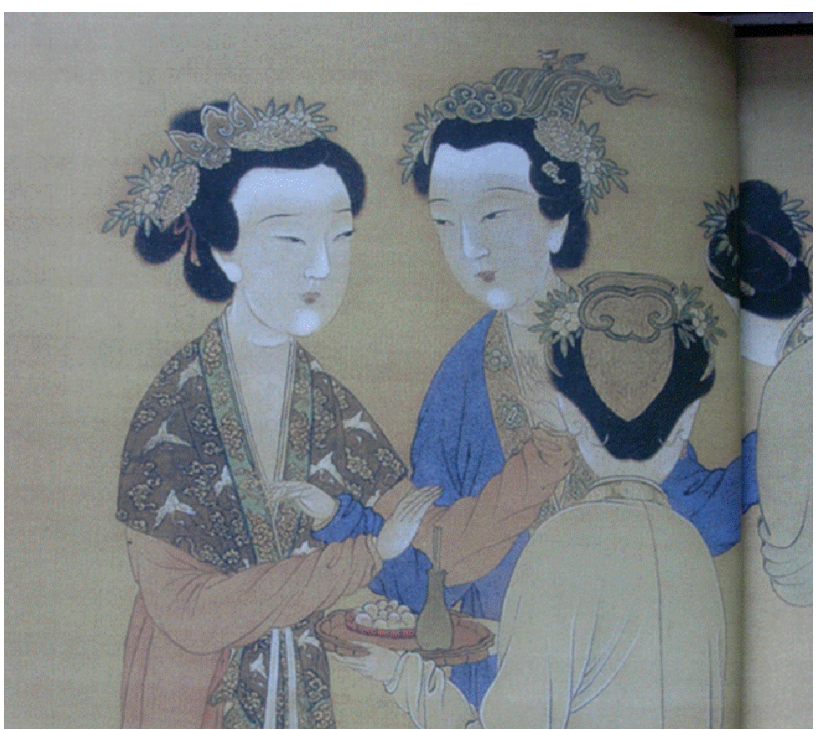

Fig. 2 Imperial Concubine Painting drawn by Bohu Tang who is one of the most famous gifted youth in Ming dynasty

by the people of Asian countries and regions. As time goes by, the most attractive upper eyelid comes out with different features than in the past. The typically modern characteristics are slim face with smooth contour, larger, almond-like eyes, Westernized double eyelid crease, even a sulcus with a large fissure, narrower distance of both eyes without epicanthus and less bulging upper eyelid. However, such typical $100 \%$ Westernized double eyelid molding once prevailed in Asian countries, and patients who accepted it may have short-term satisfaction, but soon found that they were alienated by their own national culture, and also could not be as expected-just through the same appearance, they could easily integrate into Western society, and in most Asian individuals, "Western eye" or "European eye" was not seen to be harmonious and beautiful $[1,2]$. Such features not only discard the natural eyelid features of Asians, but also are different from those of Caucasians. Because their face is an Asian face, an Asian face with Western eyes is weird and nondescriptive. So Westernization should be partial but not total, should make the finishing point to the Asian eyes. However, comparing Asian and Caucasian upper eyelids, the common sense of beauty is similar, which indicates that the wider, bigger and longer upper eye fusion is more attractive in the population. Westernized upper eyelid style with some Asian style is more attractive to the population. That is why the mean vertical palpebral height in the beautiful group is approximate to that in the Western population from this article investigation. But the essential and critical point is that Westernization of eyes should be based on and rely on the Asian characteristics. 
The article results indicate that the vertical distance of the palpebral fissure, height of the double fold, height of the lid crease and height of the closed upper eyelid were statistically significant. In our opinion, the key elements are two among the four: distance of palpebral fissure and the height of lid crease. The height of the lid crease and distance of the palpebral fissure both determine the height of the double fold. The height of the double fold is different in different cultures, even in different Asian countries. Comparing that in Korean people and Chinese people, more Chinese people may like a higher double fold. Similar results were revealed in this article. The study showed the mean eyelid creases were $5.05 \mathrm{~mm}$ in males and $5.71 \mathrm{~mm}$ in females. The average height of the double fold when eyes were closed eye was $6.6 \mathrm{~mm}$ in Korean males and $6.5 \mathrm{~mm}$ in Korean females [3]. In fact, patients do not care about the height of the double eyelid when the eyes are closed (the height of design). They only care about the width of the double eyelids when the eyes are open, that is, the size or width of the so-called double eyelid. However, the height of the double eyelid is the most important determinant of the width of the double eyelid, and also the most easily controlled factor for surgeons during the operation. It was found that the moving distance of the upper eyelid margin and the heavy thickness of the eyelid tissue are negative determinants of the height of the double eyelids (the width of double eyelid). However, in our experience, among these three factors, the height of double eyelid is the most important factor to determine the width of double eyelid, which may only be determined by the surgical designing based on where and how to mark the eyelid crease on the upper eyelid. Such upper double eyelid-plasty can still achieve good results in many clinical cases.

We totally agree that patients usually want beautiful natural double eyelids with much higher eyelid creases. The article mentioned that most patients show pictures of eyelids of beautiful actresses or beauty contestant winners when asked to show pictures of desired double eyelids. We believe it is not the average normal eyelid, but is the beautiful double eyelid, although it is much more than the minority of population. Because young patients' aesthetic ideas and philosophies are different from those of surgeons and consultants, in their mind, such beautiful actress eyes are natural. Natural definition and connotation have been greatly changed. We should open our mind to explore the modern aesthetic conception, by which only we could understand the aesthetic ideas of the double eyelids of young generations. In the meantime, physicians should stick to their own moral bottom line, aesthetic basic principles and professional knowledge, give patients pertinent suggestions, guide them to accept the correct aesthetic concept, and avoid some irremediable over-Westernized upper eyelid plastic results.

\section{Compliance with Ethical Standards}

Conflict of interest The authors declare that they have no conflict of interest to disclose.

Ethical Approval This article does not contain any studies with human participants or animals performed by any of the authors.

Informed Consent For this type of study, informed consent is not required.

\section{Reference}

1. Park S, Kim B, Shin Y (2011) Correction of superior sulcus deformity with orbital fat anatomic repositioning and fat graft applied to retro-orbicularis oculi fat for Asian eyelids. Aesthet Plast Surg 25(2):162-170

2. Sun BS (2006) Discussion on the aesthetic defects and causes of upper eyelid depression from the different characteristics of eye aesthetics in the East and the West. Chin Aesth Med 9:1040

3. Park DH, Choi WS, Yoon SH, Song CH (2008) Anthropometry of Asian eyelids by age. Plast Reconstr Surg 121:1405-1413

Publisher's Note Springer Nature remains neutral with regard to jurisdictional claims in published maps and institutional affiliations. 